\title{
Spatially periodic radio-frequency quadrupole focusing linac
}

\author{
A. A. Kolomiets" and A. S. Plastun \\ Accelerator Center, FSBI SSC RF Institute for Theoretical and Experimental Physics, \\ Moscow 117218, Russia
}

(Received 4 September 2015; published 21 December 2015)

\begin{abstract}
The new design for a spatially periodical rf quadrupole focusing linac is proposed. It consists of accelerating gaps formed between conventional cylindrical drift tubes, between drift tubes and rf quadrupoles with nonzero axial potential, and inside these rf quadrupoles, formed in the same way as in a conventional radio-frequency quadrupole (RFQ) linac with modulated electrodes. Such a combination provides both higher energy gain rate than conventional RFQ and stability of transverse motion for ion beams. The structure can be designed using various combinations of quadrupoles and drift tubes. Some options are considered in the paper using the smooth approximation method and computer simulation of beam dynamics. Transverse stability of particles has been studied. The proposed structure can provide suppression of $\mathrm{rf}$ defocusing effects on transverse beam dynamics. Some limitations of the spatially periodic rf quadrupole structure are mentioned.
\end{abstract}

DOI: 10.1103/PhysRevSTAB.18.120101

PACS numbers: 29.20.Ej, 29.27.Bd

\section{INTRODUCTION}

At the present time accelerating structures with spatially uniform radio-frequency (rf) quadrupole focusing (RFQ) are the best choice for the front end of ion linacs $[1,2,3]$. RFQ provides high efficiency bunching of injected low energy dc ion beams and their acceleration conserving initial transverse emittance.

RFQ is used for ion beam acceleration up to energies of several MeV. Acceleration for higher energies in a RFQ is inefficient due to significantly low acceleration efficiency of the structure with sinusoidal modulation of vanes compared with drift tube structures (DTL). It was shown $[4,5]$ that trapezoidal modulation of electrodes along the accelerating part of RFQ instead of sinusoidal ones increases acceleration efficiency considerably. However, RFQs are currently used only as an initial part of ion linacs due to the following reasons: (a) acceleration efficiency of the RFQ cell is still lower than drift tube structures; (b) effective shunt impedance of an RFQ becomes comparatively low at higher energies, mainly because the energy gain rate of all structures based on $\mathrm{H}$-mode cavities with constant voltage along the linac decreases as longitudinal velocity increases.

Eventually, the output energy of an RFQ is usually a compromise between the above-mentioned considerations and the optimal input energy of the subsequent accelerating structure that is usually one or other drift tube linac. For

\footnotetext{
*kolomiets@itep.ru

asplastun88@gmail.com
}

Published by the American Physical Society under the terms of the Creative Commons Attribution 3.0 License. Further distribution of this work must maintain attribution to the author $(s)$ and the published article's title, journal citation, and DOI. example, input energy of the Alvarez type structure is bounded at the bottom side by a length of quadrupole lenses placed in drift tubes. Sometimes, alternative focusing lattices (for example, FOODOO, FFDD, etc.) are applied to provide stability of transverse motion [6-10]. These modifications increase the nonlinear effects of the accelerating field on the beam dynamics. In the case of using superconducting cavities with large accelerating gradients right after RFQ, the low input energy leads to substantial coupling between transverse and longitudinal motion. Thus, increasing output energy of the front end structure is very often advisable. It is also true for the case of employment of RFQ as a separate installation.

The energy range of an $\mathrm{rf}$ quadrupole focusing can be expanded in the case of combining accelerating gaps formed by drift tubes and rf quadrupoles. Such structures with spatially periodic focusing are known for many years [11] and employed for acceleration of protons up to $30 \mathrm{MeV}$. However, they are not widespread regardless their design, manufacturing and successful operation experience as an injector for proton synchrotron in IHEP (Institute for High Energy Physics) [12]. Even the improved versions of structure [13-15] could not change this situation. It is explained by relatively complicated design, fabrication process, difficulties in rf tuning [16] and too high surface electric field on electrodes.

Nevertheless, the structures with spatially periodical rf quadrupole focusing offer the most effective way to extend the energy range of linacs with $\mathrm{rf}$ focusing providing compact and inexpensive front end sections and stand-alone linacs.

RFQ prevalence is quite ensured by well-developed resonant structures [17]. Usually different variations of 4-vane cavities are used for frequencies of several hundred 
$\mathrm{MHz}$, while heavy ion and low frequency RFQs are based mostly on 4-rod structure resonant structure.

This paper presents one more option of spatially periodical rf quadrupole structure with significantly reduced limitations that can be built on the base of the well-known resonant structures.

\section{SPATIALLY PERIODICAL FODO RF QUADRUPOLE STRUCTURE}

\section{A. General layout}

The main difference of the proposed spatially periodical rf quadrupole focusing structure from well-known ones is that it forms an accelerating field: (a) between conventional cylindrical drift tubes; (b) between drift tubes and rf quadrupoles with nonzero axial potential; (c) inside rf quadrupoles, formed in the same way as in conventional RFQ with modulated electrodes.

The structure that includes all ways of forming the accelerating field mentioned above is presented in Fig. 1. If quadrupole electrodes in the same plane have the same potential, it can produce the alternating focusing with a focusing period length $S=N \beta_{z} \lambda, N=1,3,5, \ldots, \beta_{z}=$ $v_{z} / c$ is a normalized velocity of ion beam and $\lambda$ is wavelength of the $\mathrm{rf}$ field. The case where $N=1$ and $\mathrm{rf}$ quadrupole length $L=0.5 \beta_{z} \lambda$ corresponds to spatially uniform RFQ. The focusing strength of the spatially periodical rf structure increases with the length of focusing period $S$ similar to FODO lattice of electro- or magnetostatical quadrupoles, which means that transverse stability can be provided with lower gradients in quadrupole lenses. This improvement provides more freedom in rf quadrupole focusing structure design and, in particular, allows getting more energy gain without focusing efficiency loss.

The total accelerating efficiency $T$ of the described structure depends on the particular design of drift tubes and $\mathrm{rf}$ quadrupoles. The most accurate way to calculate the accelerating efficiency of such a complicated design is to solve numerically the Laplace or Helmholtz equations. Figure 2 demonstrates the distribution of accelerating field normalized to $1 \mathrm{~V}$ along the structure shown in Fig. 1.

According to electrostatic simulations, the longitudinal component of electric potential difference at the axis between drift tubes and quadrupoles is $86 \%$ of its value

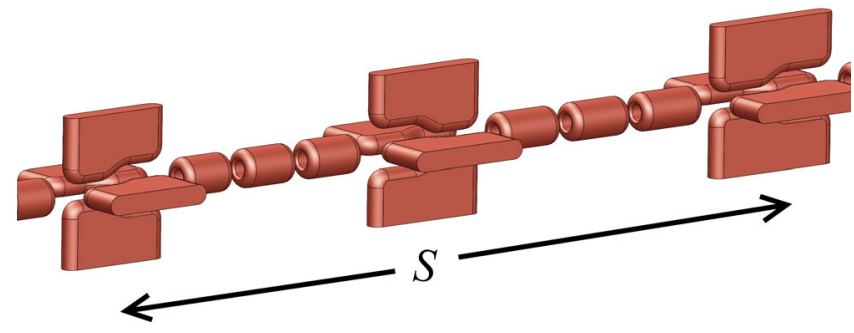

FIG. 1. Spatially periodical rf quadrupole structure; $S$-length of focusing period.

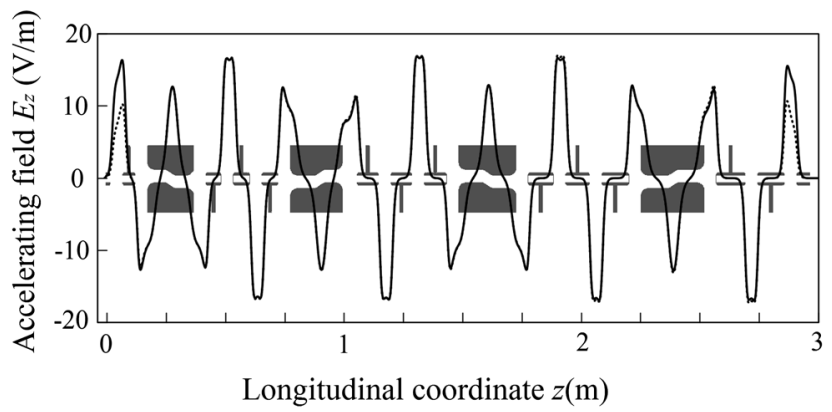

FIG. 2. Distribution of accelerating field along the lattice in front of the structure's silhouette (normalized to $1 \mathrm{~V}$ of voltage).

between two drift tubes, and the potential difference inside the quadrupole is $73 \%$ of the same value. Particle acceleration in the proposed structure takes place on each part of the lattice with a length of $\beta_{z} \lambda / 2$, whether the part is a conventional accelerating gap or a part of the quadrupole. The synchronous phase remains constant along the structure. Therefore the one little difference between various kinds of gaps is their accelerating efficiency, i.e., transit time factor. So the longitudinal beam dynamics has no differences with that one in any other drift tube linac, based on H-mode cavity and constant synchronous phase accelerating structure.

The described spatially periodic rf quadrupole focusing structure can be designed by using various combinations of quadrupoles and drift tubes. Focusing ability of the structure is obviously defined by the lattice type of the focusing period, its length and parameters of quadrupoles. In this paper we will discuss only FODO-like structures with focusing period length $S=3 \beta_{z} \lambda$ (referred to as structure B in the text) and $S=5 \beta_{z} \lambda$ (referred to as structure $\mathrm{C}$ in the text). The spatial distributions of quadrupole gradients along the axis with quadrupole length of $L=0.5 \beta_{z} \lambda$ and smooth transverse envelopes of the beam are schematically illustrated in Fig. 3. The structure A in this figure has period length of $S=\beta_{z} \lambda$ and quadrupole length of $L=0.5 \beta_{z} \lambda$. It obviously represents the conventional spatially uniform RFQ and is used below to compare with results obtained for spatially periodic structures B and C. It has to be mentioned that definition of the described focusing lattice as FODO is nominal because the real distribution of focusing and defocusing parts depends on quadrupole length and the phase of the rf field at the moment when the particle enters each quadrupole. Therefore, in this case FODO means alternation of focusing and defocusing effects from quadrupoles to the beam.

\section{B. Smooth approximation}

The basic characteristics of the rf focusing channel with FODO lattice can be obtained by the smooth approximation method [18]. The phase advance of transverse oscillations on a length of focusing period is expressed as 


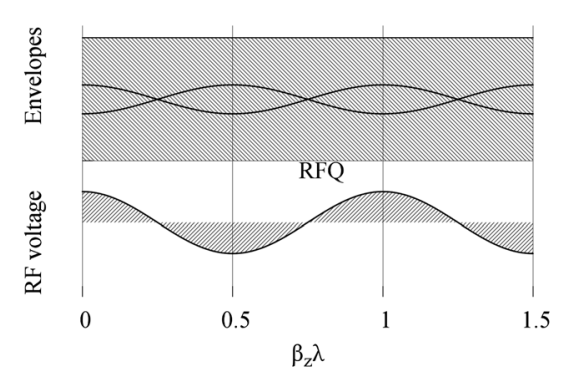

(a)

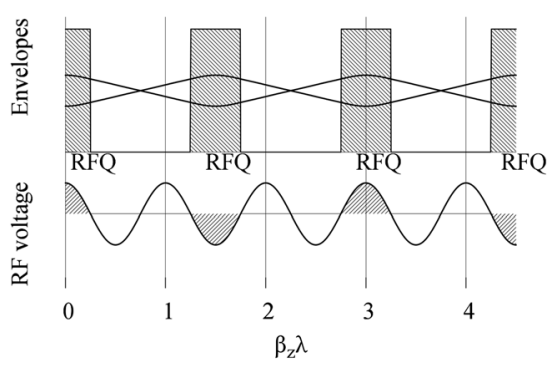

(b)

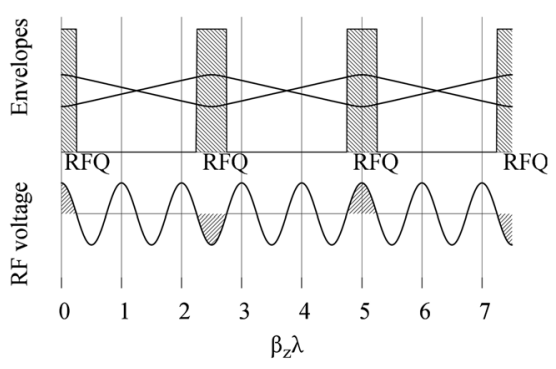

(c)

FIG. 3. Conventional RFQ (A) and spatially periodic rf quadrupole structures with FODO lattices and period lengths of $\mathrm{S}=3 \beta_{z} \lambda(\mathrm{B})$ and $\mathrm{S}=5 \beta_{z} \lambda(\mathrm{C})$.

$$
\sigma^{2}(\varphi)=\sigma_{f}^{2}(\varphi)+2 \gamma_{0} \sin \varphi_{g}
$$

where $\sigma_{f}$ is defined as phase advance $\sigma$ without defocusing effect of the accelerating field, $\gamma_{0}$ is rf defocusing parameter, $\varphi$ is the phase of the rf field at the moment when the particle is in the center of the quadrupole, $\varphi_{g}$ is the phase of the rf field at the moment when the particle is in the center of the accelerating gap. Phase advance $\sigma_{f}$ at smooth approximation can be calculated as

$$
\begin{gathered}
\sigma_{f}^{2}=\int_{\tau}^{\tau+1} q(\tau) Q(\tau) d \tau, \\
\frac{\partial^{2} q}{\partial \tau^{2}}=-Q(\tau),
\end{gathered}
$$

where $Q(\tau)$ is a periodic coefficient of the differential equation for transverse motion of a particle in a spatially periodic rf quadrupole lattice:

$$
Q(\tau)=N^{2} \lambda^{2} \frac{e}{W_{0}} G(\tau) \cos (2 \pi N \tau+\varphi),
$$

here $e$ is charge of a particle, $W_{0}$ is rest energy of a particle, $\tau$ is the normalized dimensionless variable, which gets unity at the end of focusing period $\tau=\beta_{z} c t / S$. $G(\tau)$ is distribution of the quadrupole gradient along the lattice. It is shown in Fig. 3 as square wave functions with $\varphi=0$ and $L=0.5 \beta_{z} \lambda$.

$$
S=N \beta_{z} \lambda, \quad N=1,3,5, \ldots
$$

Behavior of matched transverse envelopes presented in Fig. 3 is defined by the expression $R(\tau) \sim[1+q(\tau)]$.

The numerical estimations of the basic parameters of focusing channels shown in Fig. 3 were done assuming the parameters given in Table I. The beam parameters in Table I correspond to the output beam values of $81 \mathrm{MHz}$ RFQ [19] - the initial part of the heavy ion injector for the TeraWatt Accumulator (TWAC) facility located in the Institute for Theoretical and Experimental Physics (ITEP, Moscow). Gradients of quadrupole lenses in considered lattices were chosen in order to provide about the same phase advance of transverse particle oscillation $\sigma_{f}=60^{\circ}$.

It can be seen that the increase of the focusing period length $S$ allows the designer to use larger quadrupole apertures for the required transverse phase advance $\sigma_{f}$. Therefore, the normalized transverse acceptance of the structure remains almost unchanged.

The main intrinsic feature of rf focusing structures is the dependence of transverse motion of particles on the phase of the rf field at the moments when the particle passes

\begin{tabular}{|c|c|c|c|c|c|}
\hline \multirow[b]{2}{*}{ Parameter } & \multicolumn{5}{|c|}{ Lattice } \\
\hline & A & B & B1 & $\mathrm{C}$ & $\mathrm{C} 1$ \\
\hline Charge-to-mass ratio $Z / A$ & & & $1 / 3$ & & \\
\hline Average normalized velocity $\beta$ & & & 0.05 & & \\
\hline Frequency $f(\mathrm{MHz})$ & & & 81.36 & & \\
\hline Voltage $U(\mathrm{kV})$ & & & 300 & & \\
\hline Quadrupole aperture $R_{0}(\mathrm{~mm})$ & 12.5 & 22.0 & 22.0 & 26.4 & 26.4 \\
\hline Quadrupole length $\mathrm{L} / \beta_{z} \lambda$ & 0.5 & 0.5 & 0.72 & 0.5 & 0.8 \\
\hline Transverse phase advance $\sigma_{f}$ & $60^{\circ}$ & $33^{\circ}-75^{\circ}$ & $60^{\circ}$ & $29^{\circ}-90^{\circ}$ & $60^{\circ}$ \\
\hline Normalized transverse acceptance $A_{n}(\pi \mathrm{mm} \mathrm{mrad})$ & 28 & 22 & 30 & 17 & 26 \\
\hline
\end{tabular}
through rf quadrupoles. Figure 4 illustrates the dependence of transverse phase advance of a particle $\sigma_{f}$ calculated in smooth approximation on the phase of the rf field in the

TABLE I. Lattice and beam parameters. 


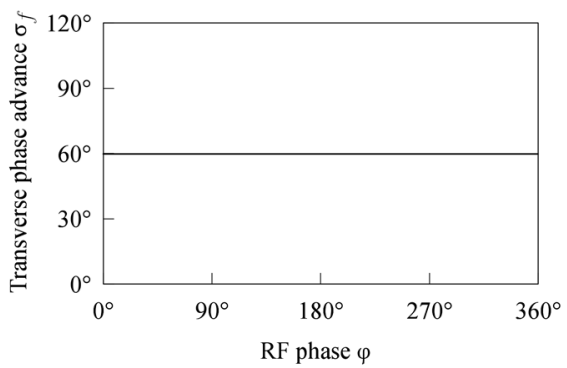

(a)

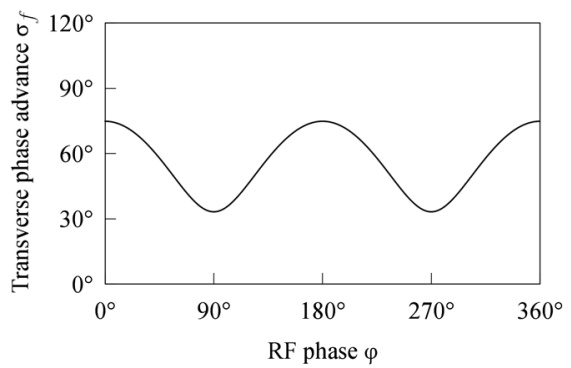

(b)

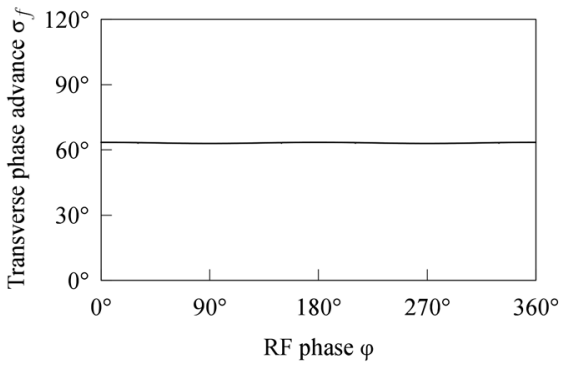

(b1)

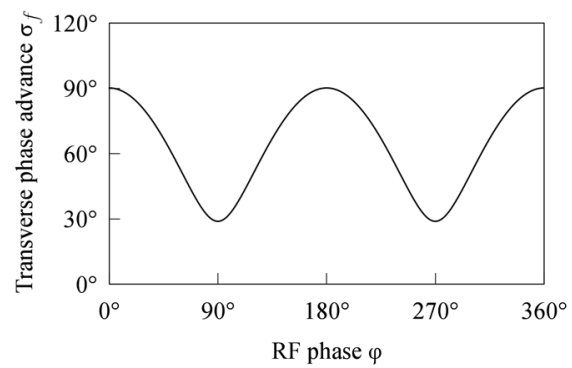

(c)

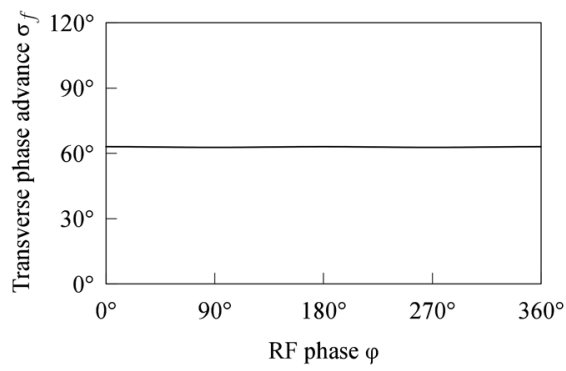

(c1)

FIG. 4. Phase advances $\sigma_{f}$ of a particle from the phase of the $\mathrm{rf}$ field in the center of the rf quadrupole. (A) $S=\beta_{z} \lambda, L=0.5 \beta_{z} \lambda$; (B) $\mathrm{S}=3 \beta_{z} \lambda, L=0.5 \beta_{z} \lambda$; (C) $\mathrm{S}=5 \beta_{z} \lambda, L=0.5 \beta_{z} \lambda$; (B1) $\mathrm{S}=3 \beta_{z} \lambda, L=0.72 \beta_{z} \lambda$; (C1) $\mathrm{S}=5 \beta_{z} \lambda, L=0.8 \beta_{z} \lambda$.

center of the rf quadrupole for $\mathrm{A}, \mathrm{B}$ and $\mathrm{C}$ lattices. Figure $4 \mathrm{~A}$ proves the fact that the phase advance $\sigma_{f}$ does not depend on the rf phase in conventional RFQ. However, structures $\mathrm{B}$ and $\mathrm{C}$ are influenced by this dependence even in the case of nonaccelerating structures. In the case of beam parameters given in Table I the variation of phase advance $\sigma_{f}$ exceeds $40^{\circ}$ for the structure $\mathrm{B}$ and $60^{\circ}$ for the structure C. It was shown that $\sigma_{f}$ variation can be suppressed by the appropriate choice of quadrupole length. Figures $4 \mathrm{~B} 1$ and $4 \mathrm{C} 1$ present phase advance $\sigma_{f}$ versus the rf phase in the center of the quadrupole for structure $\mathrm{B}$ at quadrupole length $L=0.72 \beta_{z} \lambda(\mathrm{B} 1)$ and for the structure $\mathrm{C}$ at quadrupole length $L=0.8 \beta_{z} \lambda(\mathrm{C} 1)$. This proves that the proposed spatially periodic structures can be similar to

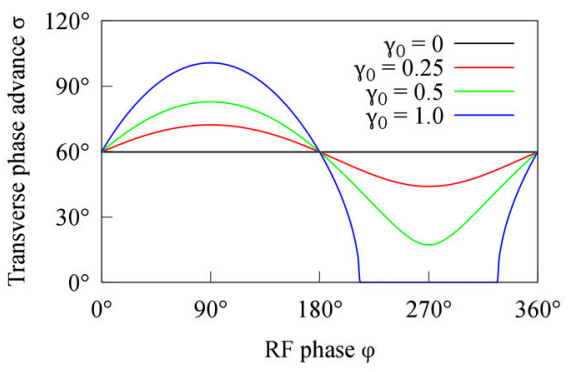

(a)

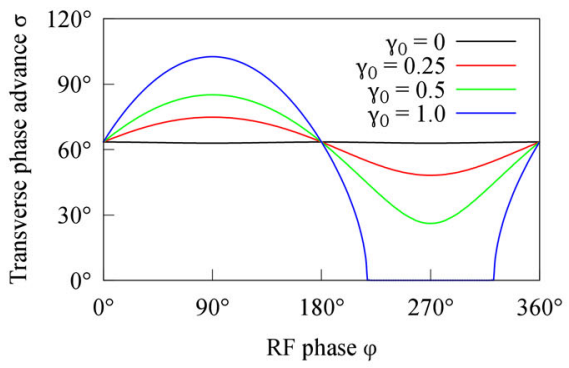

(b1)

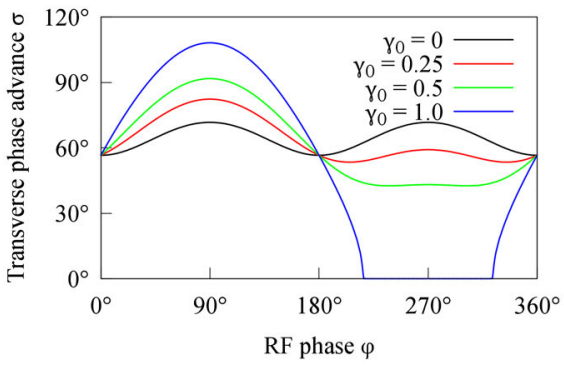

(b2)

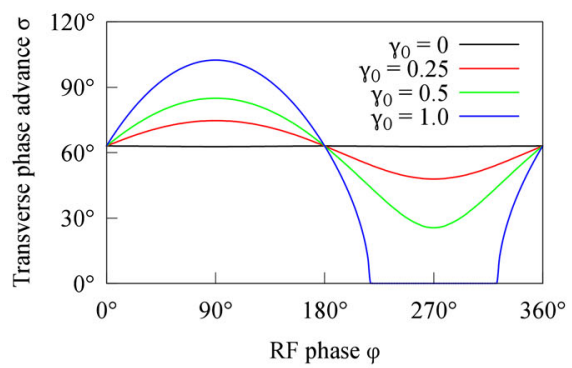

(c1)

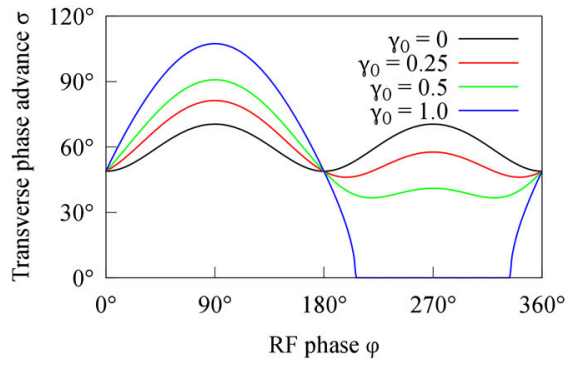

(c2)

FIG. 5. Phase advances $\sigma$ of a particle from the phase of the rf field in the center of the rf quadrupole for $\gamma_{0} \neq 0$. (A) $S=\beta_{z} \lambda$, $L=0.5 \beta_{z} \lambda$; (B1) $\mathrm{S}=3 \beta_{z} \lambda, L=0.72 \beta_{z} \lambda$; (C1) $\mathrm{S}=5 \beta_{z} \lambda, L=0.8 \beta_{z} \lambda$; (B2) $\mathrm{S}=3 \beta_{z} \lambda, L=0.8 \beta_{z} \lambda$; (C2) $\mathrm{S}=5 \beta_{z} \lambda, L=0.9 \beta_{z} \lambda$. 
conventional RFQ regarding transverse motion of beam particles. Furthermore, the independence of focusing from the $\mathrm{rf}$ phase makes the parameter choice simpler, because phases of the rf field at the moments of passing through accelerating gaps and focusing quadrupoles can be independent from each other.

The phase advance becomes dependent on the rf phase after taking into account the defocusing factor as it follows from Eq. (1). However, this effect is common for all $\mathrm{rf}$ linacs and the proposed spatially periodic structures have no difference from static quadrupole focusing structures. Plots of phase advance $\sigma$ versus the rf phase for $\gamma_{0} \neq 0$ in the case of $\varphi=\varphi_{q}$ are presented in Fig. 5.

Figures $5 \mathrm{~B} 1$ and $5 \mathrm{C} 1$ done for spatially periodic structures are similar to Fig. 5A. They demonstrate that the described spatially periodic rf focusing structure has no difference in comparison with conventional RFQ in terms of the nonlinearity of transverse motion. Moreover, it was shown that a slight increase of the quadrupole lengths $L$ suppresses the effect of $\mathrm{rf}$ defocusing on transverse motion of particles. This effect can be observed in Fig. 5B2 that corresponds to $L \approx 0.8 \beta_{z} \lambda$ and Fig. $5 \mathrm{C} 2$ that corresponds to $L \approx 0.9 \beta_{z} \lambda$.

\section{COMPUTER SIMULATION OF BEAM DYNAMICS}

\section{A. Stability chart}

In general, beam dynamics effects, caused by the coupling between longitudinal and transverse motion of particles, are increased with the length of focusing period. Eventually, it results in the limitation of longitudinal emittance of the injected beam. Figure 6 shows the stability chart (or diagram) at phase plane $(\beta, \varphi)$. The chart is plotted for structures with parameters provided in Table I and for injected particles uniformly distributed in phase range $\Delta \varphi= \pm 180^{\circ}$ with velocity spread $\Delta \beta= \pm 20 \%$. Colored

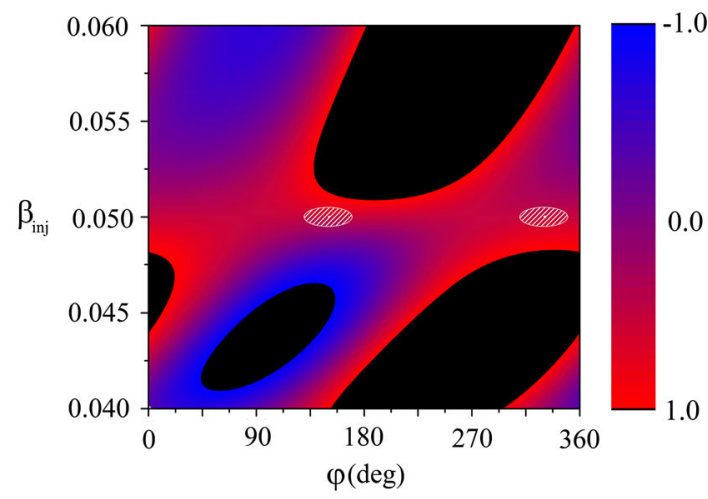

(a) areas of the plot represent stable transverse motion $(|\cos \sigma|>1)$. Black areas correspond to instability regions. Transverse phase advance $\sigma$, calculated using the method, involves construction of transfer matrices $\boldsymbol{M}$ for the focusing period from elementary matrices calculated for each single particle at each integration step of beam dynamics simulation. The criterion of stability of the transverse motion of a single particle is defined by the value of $\cos \sigma$ :

$$
\cos \sigma=\frac{\boldsymbol{M}_{11}+\boldsymbol{M}_{22}}{2}
$$

Such a stability plot for a spatially uniform RFQ looks like a uniformly colored plane, because phase advance $\sigma$ in conventional RFQ depends neither on rf phase nor on particle velocity. The velocity dispersion is absent due to the spatial uniformity of the structure and uncertainty of spatial period length definition.

White hatched ellipses in charts represent the longitudinal emittance of the beam from the initial part of the heavy ion TWAC injector. The charts show that longitudinal emittance of the injected beam is a critical parameter that influences the design of spatially periodic structures. Prints of these beams are presented in Fig. 6 as white ellipses $\left(\Delta \varphi= \pm 20^{\circ}, \Delta W= \pm 1 \%\right)$ and white points inside ellipses $\left(\Delta \varphi= \pm 1^{\circ}, \Delta W= \pm 0.1 \%\right)$. Pairs of prints on each plot correspond to two transverse planes, separated by $180^{\circ}$ in phase.

\section{B. Beam dynamics in spatially periodic rf quadrupole focusing linac}

The accurate study of beam motion in a spatially periodic rf quadrupole focusing linac can be performed with computer simulation that takes into account real 3D field distribution. Beam dynamics simulation in the structure

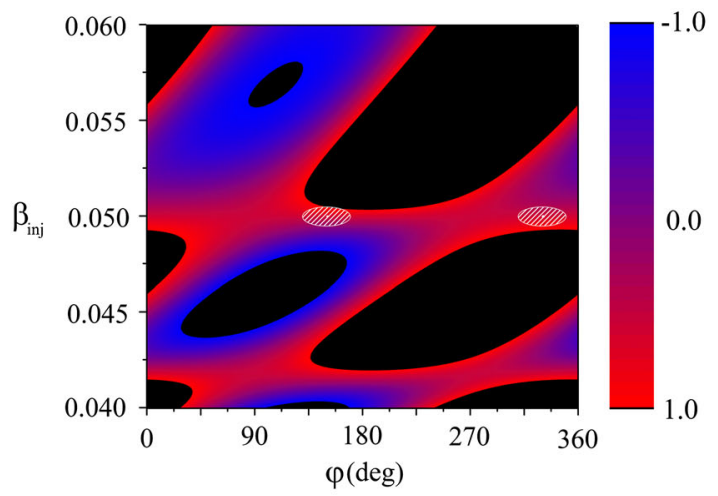

(b)

FIG. 6. Transverse stability plot on longitudinal phase space. Color represents the value of $\cos \sigma$, black represents $|\cos \sigma|>1$, (a) $S=3 \beta_{z} \lambda, L=0.72 \beta_{z} \lambda$; (b) $\mathrm{S}=5 \beta_{z} \lambda, L=0.8 \beta_{z} \lambda$. 


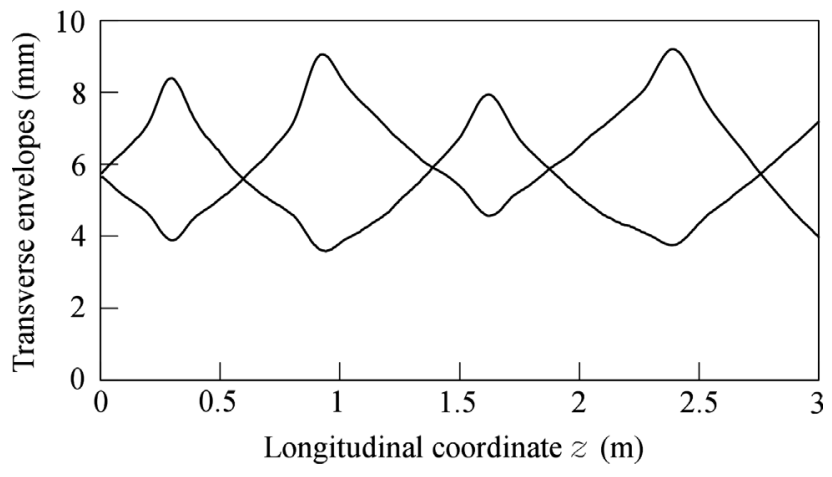

FIG. 7. Beam transverse envelopes.

that was shown in Fig. 1 and beam parameters from Table I have been done with TRANSIT code [20]. TRANSIT is a 3D macroparticle code, which uses the simplectic algorithm for integration of motion equations and the particle-in-cell (PIC) algorithm to compute the selffield of the beam. 3D distribution of the electric field in the described structure has been found by the computer simulation in electrostatic approximation.

The simulation was done for structure that includes 2.5 focusing periods with $S=5 \beta_{z} \lambda$. Total structure length is $L_{\text {str }}=3 \mathrm{~m}$, output particle energy is $W=4 \mathrm{MeV} / \mathrm{u}$. The results of beam dynamics simulation in the C-type lattice (see Table I) are presented below. Transverse envelopes of the beam are shown in Fig. 7. Output transverse and longitudinal phase-space plots of the beam are illustrated in Fig. 8. Plot (a) corresponds to the beam with small longitudinal emittance $\left(\Delta \varphi= \pm 1^{\circ}, \Delta W= \pm 0.1 \%\right)$ and "zero" current, where $W$ is a kinetic energy of particles, (b) corresponds to the beam with "zero" current and real longitudinal emittance $\left(\Delta \varphi= \pm 20^{\circ}, \Delta W= \pm 1 \%\right)$, and (c) corresponds to the beam with real longitudinal
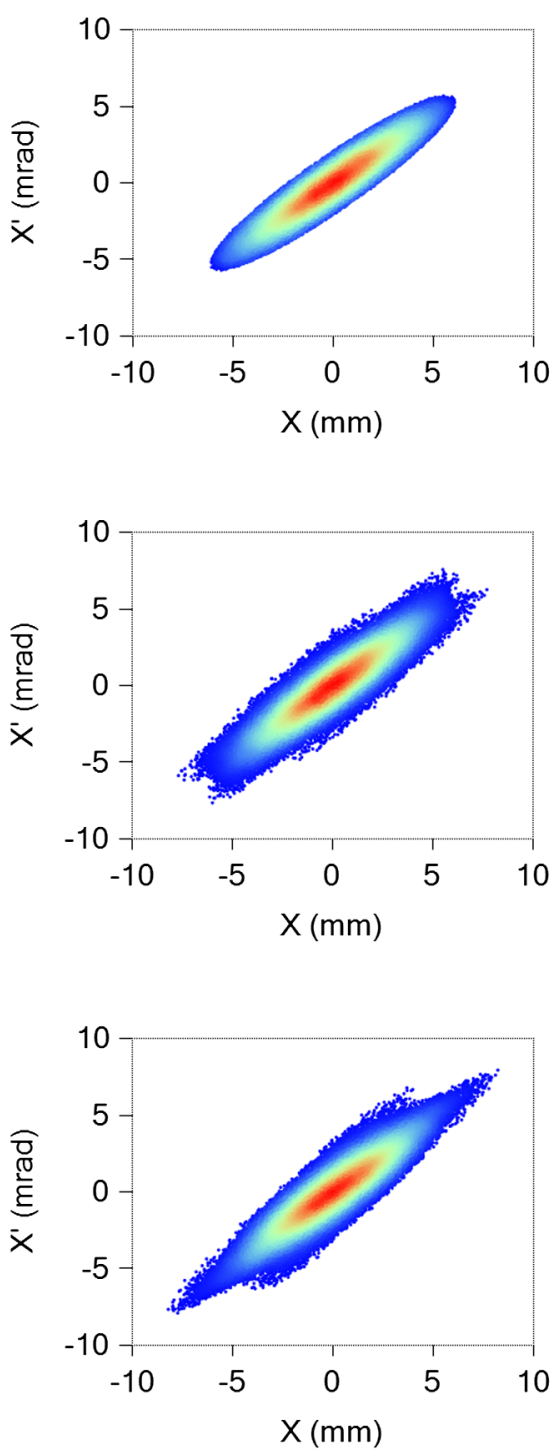

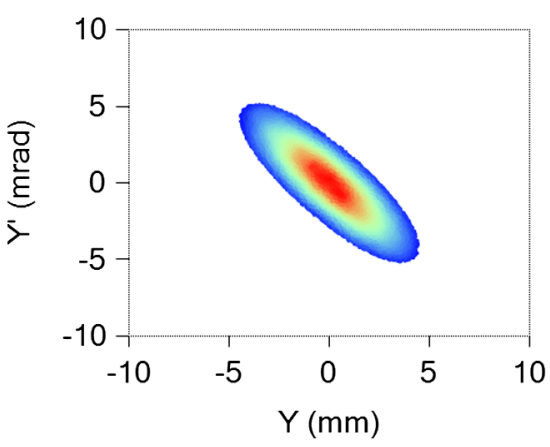

(a)

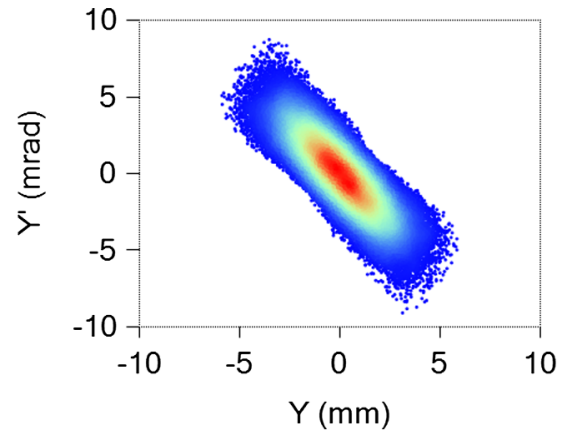

(b)

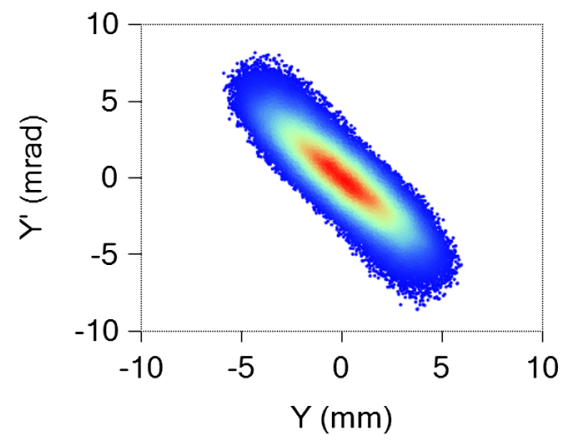

(c)
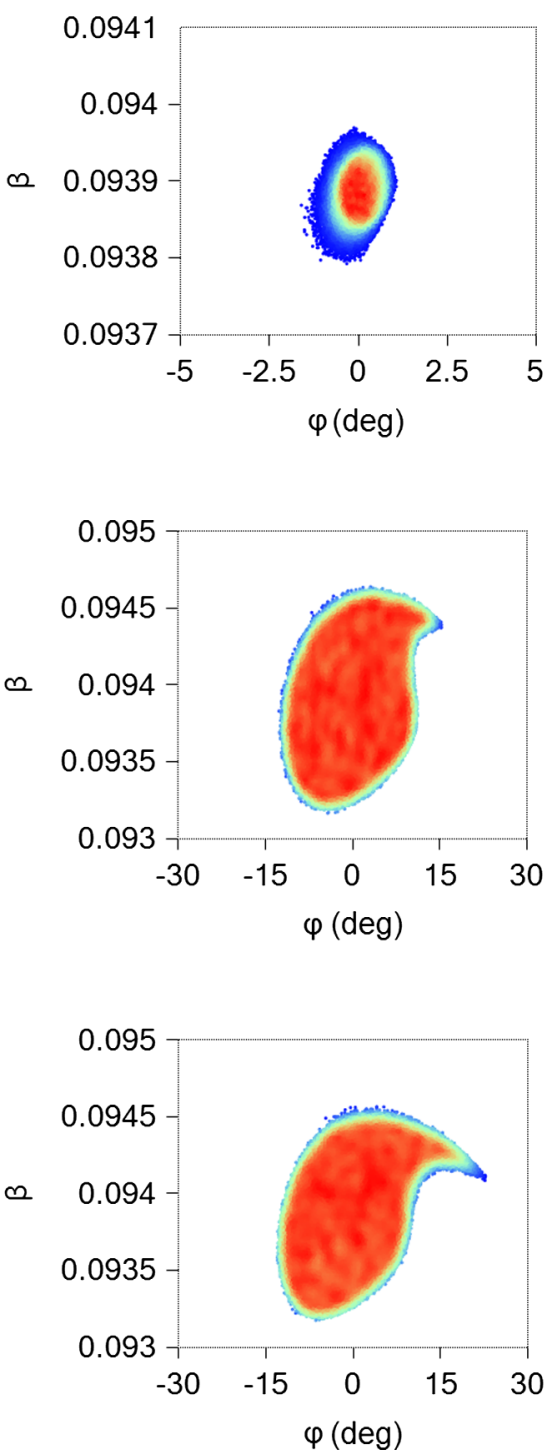

FIG. 8. Phase-space plots of output beam. 
TABLE II. Root-mean-square emittance growth.

\begin{tabular}{lccc}
\hline \hline & $\mathrm{a}$ & $\mathrm{b}$ & $\mathrm{c}$ \\
\hline Plane $\left(X X^{\prime}\right)$ & 1.004 & 1.138 & 1.144 \\
Plane $\left(Y Y^{\prime}\right)$ & 1.005 & 1.170 & 1.229 \\
Plane $(\varphi W)$ & 1 & 1.016 & 1.032 \\
\hline \hline
\end{tabular}

emittance $\left(\Delta \varphi= \pm 20^{\circ}, \Delta W= \pm 1 \%\right)$ and beam current of $20 \mathrm{~mA}$.

Transverse rms emittance growth can be used as a measure of nonlinearity of beam motion. Values of emittance growth obtained with computer simulation are presented in Table II. The data in Table II confirm that longitudinal emittance is the main cause of transverse emittance growth.

Simulation results show that for small longitudinal emittance of the injected beam and for zero beam current there is no transverse emittance growth. As would be expected, considerable emittance growth appears for

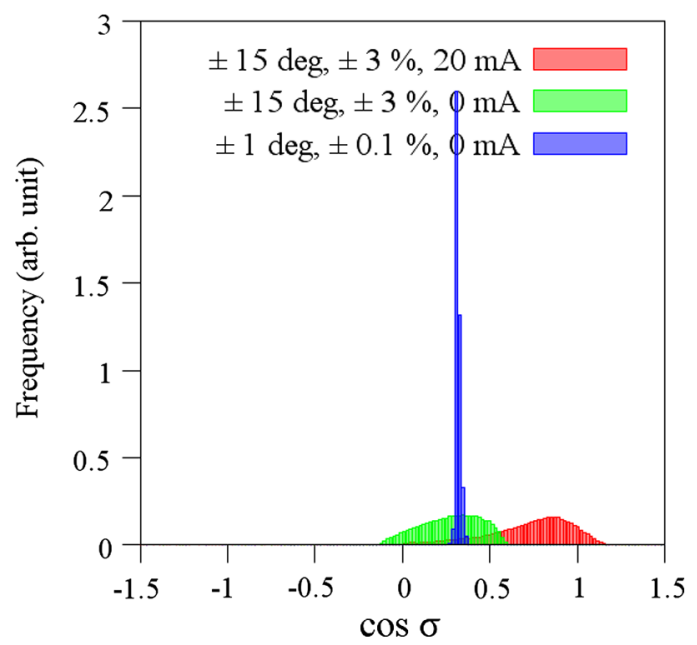

(a)

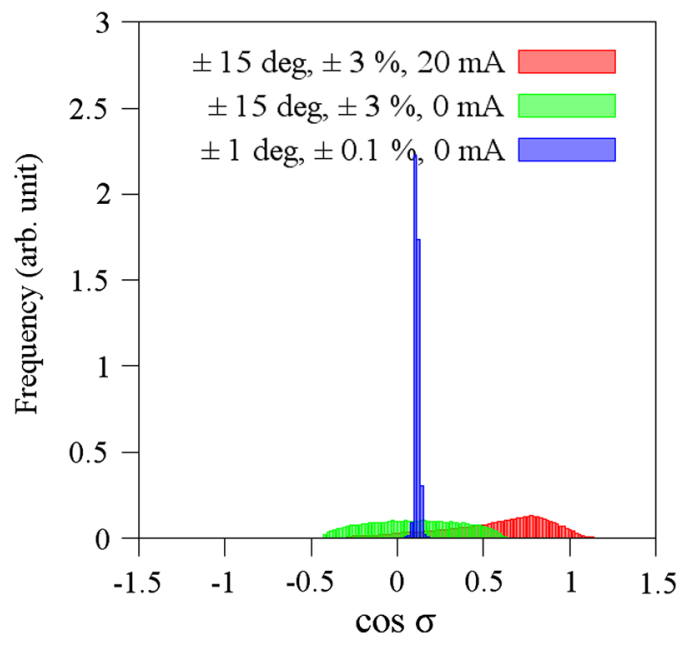

(b)

FIG. 9. Histogram for transverse phase advance of particles in a beam: (a) X-plane, (b) Y-plane. longitudinal emittance provided by preceding RFQ. In this case transverse emittance growth is about $17 \%$ for zero beam current. Consideration of $20 \mathrm{~mA}$ beam current increases transverse emittance up to $23 \%$.

TRANSIT code has the option that allows more detailed study of transverse motion stability of the beam. The option is based on the same matrix method used to plot stability charts. The results of such analysis are presented in histograms in Fig. 9. The histograms show the particle distribution of $\sigma$. Blue histograms correspond to low longitudinal emittance of the injected beam with zero current. All particles are in the stable region and there is only a small dispersion of transverse phase advance of single particles. Green histograms correspond to the realistic value of longitudinal emittance. The mean value of the histogram is the same as in the previous case but considerable dispersion of transverse phase advances of single particles is now present. The most important fact is that some particles are outside of the stability region. The presence of local instability is the reason of transverse emittance growth observed in Fig. 8. Red histograms demonstrate the shift of mean value due to Coulomb tune depression and a bit larger dispersion of phase advances. The histograms illustrate the effect of nonlinear external field or self-field of the beam on transverse dynamics. The calculated dispersion can be taken as a criterion for nonlinearity of beam motion. Regardless of nonlinearities the proposed structure provides a high energy gain rate of $2.5 \mathrm{MeV} / \mathrm{m}$ with moderate transverse emittance growth even for $S=5 \beta_{z} \lambda$.

\section{CAVITY}

An important feature of the proposed structure is that it can be built on the base of the same resonant structure as the preceding conventional RFQ. In particular, the second accelerating section for the TWAC injector can be constructed on the base of the conventional 4-vane cavity resonator. Radio-frequency quadrupole lenses are formed

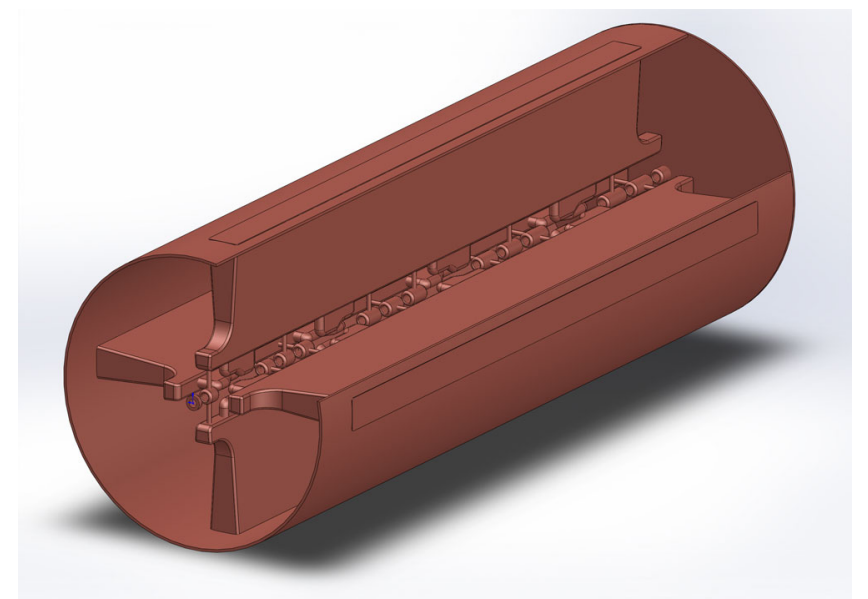

FIG. 10. 4-vane cavity resonator of a spatially periodic rf quadrupole linac. 
TABLE III. Main parameters of the linac.

\begin{tabular}{lc}
\hline \hline Parameter & Value \\
\hline Charge to mass ratio $Z / A$ & $1 / 3$ \\
Energy range $(\mathrm{MeV} / \mathrm{u})$ & $1.6-4.1$ \\
Resonator diameter $D_{R}(\mathrm{~mm})$ & 1050 \\
Resonator length $L_{R}(\mathrm{~mm})$ & 2960 \\
Voltage $U(\mathrm{kV})$ & 475 \\
Operating frequency $f(\mathrm{MHz})$ & 81.36 \\
Quality $Q$ & 23700 \\
Pulsed rf power consumption $P_{R F}(\mathrm{~kW})$ & 490 \\
Beam power gain $(20 \mathrm{~mA}) P_{B}(\mathrm{~kW})$ & 150 \\
Total power consumption $P(\mathrm{~kW})$ & 640 \\
Effective shunt impedance $Z T^{2}(\mathrm{M} \Omega / \mathrm{m})$ & 40 \\
\hline \hline
\end{tabular}

in the same manner as in conventional RFQ and drift tubes are placed between opposite vanes. Drift tubes make the opposite vanes electrically shorted and thus provide suppression of the lowest dipole modes of the cavity. The computer model of such a cavity is presented in Fig. 10. The main parameters of the spatially periodic rf quadrupole linac, based on this cavity, are shown in Table III.

\section{SUMMARY}

The new option of a spatially periodic rf quadrupole structure is proposed. Accelerating and focusing fields are formed by combinations of drift tubes and rf quadrupoles on a large focusing period length. It provides the unique freedom for lattice parameters and can be used, for example, to maintain required focusing strength in a heavy ion linac or to achieve high energy gain rate.

The computer study of the structure demonstrated that in the case of a wise choice of rf lens lengths the beam dynamics is similar to a conventional accelerating structure with static quadrupole focusing. The main factors that influence transverse dynamics and lead to transverse emittance growth are longitudinal emittance of an input beam and acceleration rate of the structure.

A spatially periodic rf quadrupole linac with the proposed structure can be based on the same cavity as an RFQ, and provides high energy gain rate and high shunt impedance with moderate transverse emittance growth due to an effective combination of drift tubes and $\mathrm{rf}$ quadrupoles. The proposed option of a spatially periodic rf focusing structure can be useful for the design of front ends of ion linacs for acceleration of all ion species up to several tens $\mathrm{MeV}$ energies as well as for design of compact and relatively inexpensive ion linacs for this energy range.
[1] I. M. Kapchinsky and V. A. Teplyakov, Prib. Tekh. Eksp. 2, 19 (1970).

[2] M. Vretenar et al., in Proceedings of NAPAC 2013, Pasadena, CA, USA (NAPAC, Pasadena, 2013), pp. 52-56.

[3] S. V. Kutsaev, B. Mustapha, P. N. Ostroumov, A. Barcikowski, D. Schrage, J. Rodnizki, and D. Berkovits Phys. Rev. ST Accel. Beams 17, 072001 (2014).

[4] O. K. Belyaev et al., in Proceedings of the 20th International Linac Conference, LINAC-2000, Monterey, CA, 2000 (SLAC, Menlo Park, CA, 2000), pp. 259-261.

[5] P. N. Ostroumov et al., Phys. Rev. ST Accel. Beams 15, 110101 (2012).

[6] I. M. Kapchynskij and V. K. Plotnikov, Prib. Tekh. Eksp. 5, 12 (1967).

[7] D. J. Warner, in Proceedings of LINAC 1988, Williamsburg, Virginia, USA, (LINAC, Williamsburg, VA, 1988) pp. 109-111.

[8] M. Vretenar and D. J. Warner, in Proceedings of LINAC 1990, Albuquerque, New Mexico, USA, (LINAC, Albuquerque, NM, 1990) pp. 96-98.

[9] S. Ramberger, A. M. Lombardi, M. Vretenar et al., in Proceedings of the 25th International Linear Accelerator Conference, LINAC-2010, Tsukuba, Japan (KEK, Tsukuba, Japan, 2010), pp. 560-562.

[10] M. Comunian, F. Grespan, A. Pisent et al., in Proceedings of LINAC 2012, Tel-Aviv, Israel, (LINA, Tel-Aviv, Israel, 2012) pp. 918-920.

[11] Yu. A. Budanov, O. K. Belyaev, S. V. Ivanov et al., in Proceedings of LINAC 2004, Lübeck, Germany, (LINAC, Lübeck, Germany, 2004) pp. 285-287.

[12] V. A. Zenin, A. P. Maltsev, V. A. Teplyakov et al., in Proceedings of the 17th International Linear Accelerator Conference (LINAC-1994), Tsukuba, Japan, 1994 (KEK, Tsukuba, Japan, 1994), pp. 158-162.

[13] D. A. Swenson, in Proceedings of the 17th International Linear Accelerator Conference (LINAC-1994), Tsukuba, Japan, 1994 (Ref. [12]), pp. 804-806.

[14] S. Minaev, Nucl. Instrum. Methods Phys. Res., Sect. A 489, 45 (2002).

[15] A. I. Balabin and G. N. Kropachev, Nucl. Instrum. Methods Phys. Res., Sect. A 459, 87 (2001).

[16] O. K. Belyaev et al., in Proceedings of the 17th International Linear Accelerator Conference (LINAC1994), Tsukuba, Japan, 1994 (Ref. [12]), pp. 296-298.

[17] H. Klein, in Proceedings of PAC 1983, Santa Fe, New Mexico, USA, (PAC, Santa Fe, New Mexico, 1983), pp. 3313-3322.

[18] I. M. Kapchinsky, Theory of Resonance Linear Accelerators (Harwood, Chur, Switzerland, 1985).

[19] V. Andreev, N. N. Alexeev, A. Kolomiets et al., in Proceedings of the 2nd International Particle Accelerator Conference, San Sebastián, Spain (IPAC-2011, Spain, 2011), pp. 2622-2624.

[20] A. A. Kolomiets, A. S. Plastun, and T. E. Tretyakova, in Proceedings of RuPAC 2014, Obninsk, Kaluga Region, Russia, (RuPAC, Obninsk, Kaluga Region, 2014), pp. 51-53. 